\title{
Digitale Transformation in Krankenhäusern: Potenziale und Innovationen entlang des stationären Leistungsprozesses
}

\section{Zusammenfassung}

Stationäre Einrichtungen stehen, bedingt durch die Asymmetrie zwischen erhöhtem Bedarf bei nur begrenzten Kapazitäten, einer Vielzahl an Herausforderungen entgegen. Zeitmangel, Überlastung und Prozessineffizienzen bedingen risikobehaftetes Verhalten der Leistungserbringer, was zu Lasten der Patientensicherheit gehen kann. Vor diesem Hintergrund ist die Entwicklung innovativer Bewältigungsstrategien unabdinglich, um die patientenzentrierte Versorgung wieder in den Fokus zu rücken. Digitale Innovationen versprechen, insbesondere im Bereich der Unterstützungsprozesse, zahlreiche Möglichkeiten, um den Konflikt zwischen verfügbaren Ressourcen und gestiegenen Anforderungen bewältigen zu können (Deiters et al. 2018). Durch gezielten Einsatz digitaler Technologien in jedem Segment des stationären Leistungsprozesses kann es gelingen, prozessuale Nutzeneinbußen auszugleichen und durch Vernetzung der einzelnen Segmente, in verbesserter Versorgungsqualität, einen Mehrwert für alle Beteiligten zu schaffen.

\section{Inhalt}

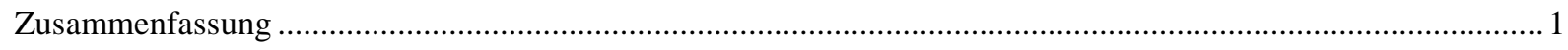

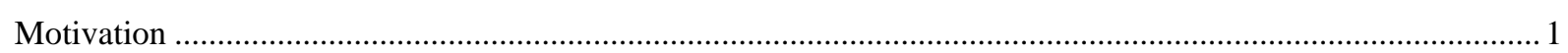

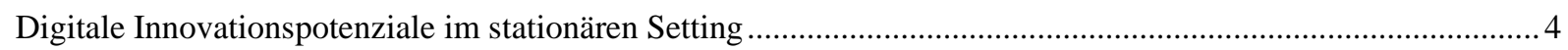

Prozessstrukturen in stationären Versorgungseinrichtungen..............................................................

Potenziale durch technologische Innovationen entlang des stationären Leistungsprozesses ............................. 8

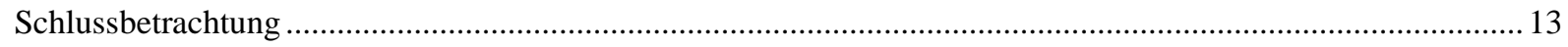

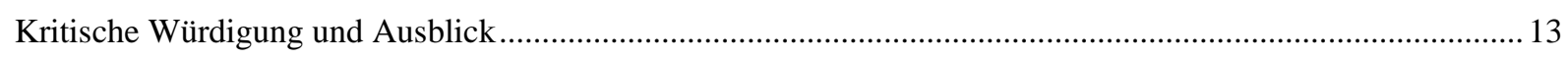

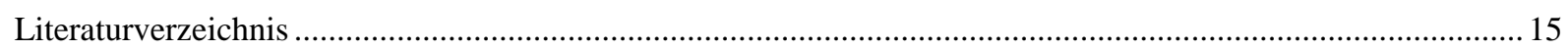

\section{Motivation}

Das deutsche Gesundheitssystem ist von einer vorherrschenden, innovationshemmenden Struktur und finanzieller Dysbalance geprägt (Deiters et al. 2018; Rynning 2008). Insbesondere Krankenhäuser als zentral zu vernetzende Einrichtungen des Gesundheitswesens stehen innerhalb der Branche im Mittelpunkt des Interesses (Di Vincenzo 2018). Zum derzeitigen Stand weisen Krankenhäuser einen Investitionsstau von rund 5,6 Milliarden Euro auf und beeinträchtigen damit die bestehende Infrastruktur, Prozesse und erbrachte Dienstleistungen (Deloitte 2018). Nicht-gewinnorientierte Krankenhäuser können nur begrenzt auf Fremdkapital zurückgreifen und auch Fördermittel aus öffentlicher Hand sind knapp bemessen (Augurzky und Beivers 2014), was die Optimierung von Betriebsabläufen und Versorgungsinnovationen erschwert. Mussten vor der Einführung des Gesundheitsstrukturgesetzes 1993 noch die Kostenträger die Verantwortung für die retrospektive Vergütungssystematik des Selbstkostendeckungsprinzips tragen, liegt es seit Einführung der pauschalierten 
Vergütung in der Eigenverantwortung der Krankenhäuser, die DRG-Fallpauschale gewinnbringend umzusetzen (Rau et al. 2009). Nach Goedereis (2009) sind Krankenhäuser im Zuge dessen in der Vergangenheit bestrebt gewesen, Kostenreduktionen vorzunehmen. Da Krankenhausleistungen mit einem Anteil von durchschnittlich zwei Dritteln der Gesamtkosten für Personalkosten sehr personalintensive Dienstleistungen darstellen, standen diese, mit schwerwiegenden Folgen für den Versorgungsalltag, häufig im Mittelpunkt von Kostensenkungsprogrammen (Simon 2014). Darüber hinaus beeinträchtig die sektorale Trennung der ambulanten und stationären Versorgung die Vernetzung der zahlreichen der an der Behandlung beteiligten Professionen. Die vorliegende Fragmentierung führt zu erheblichen Schnittstellenproblemen und Versorgungsbrüchen, mit daraus resultierenden Ineffizienzen und Mängeln in der Behandlungsqualität (Gerlinger 2013).

Den infrastrukturellen Herausforderungen steht derweil eine, durch die Bedarfe einer zunehmend alternden Gesellschaft, drastisch steigende Nachfrage nach medizinischen Dienstleistungen und Ressourcen entgegen. Die steigende Nachfrage findet ihren Ursprung in erster Linie in der steigenden Lebenserwartung bei gleichzeitigem Abfall der Geburtenrate (van Baal et al. 2018). Gravierende Veränderungen des Versorgungsbedarfes bringt auch die nachgewiesene Expansion der Lebensjahre mit Multimorbidität, parallel zur steigenden Lebenserwartung, mit sich (Beard und Bloom 2015; Tetzlaff et al. 2017). Dieser Wandel wird die Zahl der Patienten in den nächsten Jahren erhöhen, so dass schätzungsweise weitere 30.000 Vollzeitkräfte in deutschen Krankenhäusern benötigt werden (Springer Medizin Pflegezeitschrift 2018). Dabei sinkt zusätzlich die Zahl der Menschen im erwerbsfähigen Alter zwischen 20 und 65 Jahren kontinuierlich, weswegen der Fachkräftemangel deutlich zunimmt und eine Vorhaltung ausreichender Personalkapazitäten für die Bewältigung des Leistungsvolumens nur schwer möglich ist (Deloitte 2018).

Während die Versorgungsqualität stets im Mittelpunkt medizinischer Leistungserbringung stehen sollte, kann diese Prämisse unter den gegebenen Umständen jedoch nur eingeschränkt erfüllt werden. Die entstehende Asymmetrie zwischen erhöhtem Bedarf bei limitierten Kapazitäten führt zu einer mangelhaften Ausführung der zugrundeliegenden Tätigkeiten, da die beteiligten Akteure dem auftretenden Arbeitsvolumen nicht vollumfänglich nachkommen können (Weissman et al. 2007). Dieser Mangel kann zur impliziten Rationierung von Leistungen führen und die Versorgungsqualität erheblich beeinträchtigen. Für Patienten und medizinisches Personal bedeutet Rationierung eine Veränderung des Versorgungsalltags und eine ungewollte Abwendung von etablierten, patientenzentrierten Pflegeleitbildern. Bedingt durch den auftretenden Zeitmangel und die Überforderung der Fachkräfte kann es zur Vernachlässigung von Patienten sowie administrativer Aufgaben, wie Dokumentationen oder Pflegeübergaben, kommen (Hufnagl et al. 2019). Die Fehleranfälligkeit von Informationen steigt, was Doppeluntersuchungen begünstigt und Verwechslungen von beispielsweise Patienten oder Medikationen, mit daraus resultierenden risikobehafteten Behandlungsfehlern, zur Folge haben kann. Dabei erschwert die Koordination der auf Station beteiligten Professionen die Fokussierung des medizinischen Fachpersonals auf die originären Tätigkeiten. In der Realität werden eine Vielzahl der Unterstützungsprozesse durch pflegerisch ausgebildetes Fachpersonal erbracht, da die Abstimmung mit den dafür vorhergesehenen Berufsgruppen häufig zeitintensiver ist, als die selbstständige Erledigung der Aufgabe. Nach Weiß (2014) geschieht es häufig, dass das Pflegepersonal Tätigkeiten unterhalb des eigentlichen Qualifikationsniveaus nachgeht, was eine Fehlallokation der ohnehin nur in mangelnder Besetzung vorhandenen Fachkräfte darstellt.

Vor diesem Hintergrund ist die Entwicklung innovativer Bewältigungsstrategien unabdinglich, um die patientenzentrierte Versorgung wieder in den Fokus zu rücken. Es bliebe den Versorgern innerhalb des gegebenen 
Spannungsfeldes die Möglichkeit, beispielsweise durch die bewusste Einschränkung des angebotenen Leistungsportfolios im Sinne einer Spezialisierung, die Nachfrage zu kontrollieren und an die gegebenen Ressourcen anzupassen. Diese Herangehensweise ist kritisch zu bewerten, da eine Veränderung des Leistungsspektrums dazu führen kann, dass Krankenhäuser nicht mehr die Voraussetzung zur Aufnahme in den Krankenhausplan der Länder erfüllen. Art. 5 Abs. 2 Satz 2 BayKrG regelt beispielsweise, dass die Aufnahme eines Hauses in den Krankenhausplan ganz oder hinsichtlich bestimmter Versorgungsaufgaben widerrufen werden kann, sofern die Voraussetzungen nicht, beziehungsweise nur vorübergehend nicht, mehr vorliegen. Darüber hinaus bestünde auch die Möglichkeit, die benötigten Personalressourcen sowie die Ausstattung aufzustocken, um der Veränderung des Versorgungsbedarfes nachzukommen. Dies ist jedoch bei gegebener Budgetierung und fehlenden Investitionsmitteln nur beschränkt umsetzbar.

Um den Veränderungen des Versorgungsalltags entgegenzuwirken, müssen die nur im begrenztem Maße zur Verfügung stehenden Mittel und Fachkräfte effizient eingesetzt werden (Hufnagl et al. 2019). Den beschriebenen Beeinträchtigungen des medizinischen Versorgungsalltags, Zeitmangel und Überforderung sowie die damit verbundene Fehleranfälligkeit, muss zielgerichtet entgegnet werden. Da etablierte Bewältigungsansätze nur in begrenztem Maße zielführend sind, bedarf es der Konzeption innovativer Strategien, die die Neuausrichtung der Prozessebene zum Inhalt haben. Wie Lohmann (2009) beschreibt, erwarten Patienten eine erstklassige medizinische Versorgung, die daher im Fokus der Prozesse stehen muss. Dienstleistungen resultieren aus den vorgefundenen Rahmenbedingungen. Eine Verbesserung im Hinblick auf Qualität und Wirtschaftlichkeit ist somit auf einen tiefgreifenden Paradigmenwechsel von einer zufälligen zu einer strukturierten und standardisierten Arbeitsweise angewiesen. Voraussetzung dafür ist eine kritische Auseinandersetzung mit den bestehenden Organisations- und Prozessstrukturen, damit eine Optimierung der Prozessabläufe zur reibungslosen Ausführung der Kerntätigkeiten erfolgen kann. Die Minimierung unnötiger Tätigkeiten (Verschwendung) zielt auf die Steigerung der Prozesseffizienz ab, was die Aspekte der Versorgungsqualität und Patientensicherheit positiv bedingt (Traeger 2013). Als Verschwendung zählen sämtliche Tätigkeiten und Prozessbestandteile, welche keinen Beitrag zur Wertschöpfung leisten und demnach keinen Nutzen aus Sicht des Kunden (Patient oder Kostenträger) stiften (Bergmann und Lacker 2009).

Aus gesamtorganisatorischer Perspektive dient diese Leistungsverbesserung dazu, dem Unternehmen eine nachhaltige Position als Dienstleister mit hoher Ergebnisqualität im umkämpften Krankenhausmarkt zu sichern. Die alleinige Neugestaltung zugrundeliegender Prozesse reicht jedoch nicht aus, um den Anforderungen entgegen zu treten. Dazu bedarf es der technologischen Unterstützung der Mitarbeiter in nicht-medizinischen Belangen. Digitale Innovationen entlang unterstützender Prozesse versprechen zahlreiche Möglichkeiten, um den Konflikt zwischen verfügbaren Ressourcen und gestiegenen Anforderungen bewältigen zu können (Deiters et al. 2018). Dabei fördern Prozessinnovationen nicht nur die Wirtschaftlichkeit und Wettbewerbsfähigkeit durch Effizienzsteigerungen, vielmehr richtet dies den Fokus auf den originären Versorgungsauftrag der Einrichtungen, die patientenzentrierte Erbringung medizinischer Dienstleistung. Die Verringerung unerwünschter Ereignisse und die Hebung prozessualer Reserven kann, beispielsweise durch eine einhergehende Senkung der Verweildauer, eine simultane Reduktion der Fallkosten mit sich führen. Die Wertschöpfung wird in Dienstleistungsunternehmen, wie sie auch Krankenhäuser darstellen, durch die Mitarbeiter generiert und lässt sich aufgrund des Vertrauensverhältnisses zwischen Personal und Patient nicht ohne Weiteres digitalisieren (Riepe und Schwanenflügel 2013). 


\section{Digitale Innovationspotenziale im stationären Setting}

Der Durchbruch von Informations- und Kommunikationstechnologien hat einen nachhaltigen Einfluss auf die Fundamente von Arbeit und Wirtschaft sowie des gesellschaftlichen Zusammenlebens. Die Nutzung von Technologien zur Gestaltung effizienter Geschäftsprozesse findet nicht nur im produzierenden Gewerbe, sondern auch im Dienstleistungsbereich zunehmend Anwendung. Die digitale Transformation wird im Gesundheitswesen allerdings nur langsam vollzogen. So bildet das Gesundheitswesen mit 37/100 Punkten das Schlusslicht im Vergleich des Wirtschaftsindex DIGITAL, eine Kennzahl, die den branchenspezifischen Fortschritt der Digitalisierung von Unternehmen misst (Bundesministerium für Wirtschaft und Energie 2018). Obgleich die Bundesregierung bereits im Jahre 2003 mit dem Gesetz zur Modernisierung der gesetzlichen Krankenversicherung die Entwicklung der elektronischen Gesundheitskarte durch die Selbstverwaltungskörperschaften, organisiert in der gemeinsamen Gesellschaft der Gematik, beschlossen hat, erfolgte die Umsetzung aufgrund starken Gegenwindes von Seiten der Ärzte und Krankenkassen nur schleppend (Tebroke 2017). Demzufolge wurde 2015 das E-Health-Gesetz verabschiedet, welches einen Zeitrahmen für die Einführung setzt und weitere Anreize zur Digitalisierung bietet. Durch den Einsatz von Informationstechnologie, die intelligente Integration behandlungsrelevanter Daten und Anwendungssysteme sowie die Berücksichtigung der Interessen beteiligter Akteure können die Treiber der Digitalisierung zur Unterstützung der Professionen und der Steigerung der Wertschöpfung genutzt werden (Georgantzas und Katsamakas 2008). Schätzungen zufolge ist die Aufgabenlast medizinischen und pflegerischen Personals um rund ein Drittel reduzierbar, etwa durch den Transfer der Aufgaben auf andere Berufsgruppen. Eine zukunftsorientierte Möglichkeit zur Reduktion der Aufgabenlast und damit Erhöhung der Versorgungsqualität liegt in der Optimierung der zugrundeliegenden Prozesse durch die Nutzung digitaler Innovationen. In Krankenhäusern werden Digitalisierungsvorhaben vielfach auf die medizinische Dokumentation reduziert, diese Vereinfachung wird jedoch dem generell notwendigen Wandel der Branche nicht gerecht. Es ist von zentraler Bedeutung für die Wettbewerbsfähigkeit von Unternehmen, technologische Unterstützung möglichst effizient in die täglichen Prozessabläufe einzubinden, damit sämtliche Stakeholder erfolgreich und ohne Beeinträchtigung kooperieren können (Deiters et al. 2018). Insbesondere im Bereich der Prozessharmonisierung und -digitalisierung verspricht die Unterstützung durch Informationssysteme eine enorme Qualitätsverbesserung bei gleichzeitiger Reduktion der Kosten (Denner et al. 2018). Voraussetzung für eine technologische Unterstützung ist jedoch, dass Prozesse, beispielsweise auf Station oder in Funktionsbereichen, bereits einen hohen Reifegrad und eine exakte Definition aufweisen. Die reine Digitalisierung bereits bestehender, jedoch nicht-wertschöpfungsorientierter Prozesse bietet nur geringen Mehrwert, da „schlechte“ analoge Prozesse durch Digitalisierung lediglich zu „schlechten“ digitalisierten Prozessen transformiert werden. Es bedarf der vorangestellten Optimierung dieser und der Ausrichtung auf die gesamtorganisatorische Wertschöpfung.

\section{Prozessstrukturen in stationären Versorgungseinrichtungen}

Betrachtet man die klinische Leistungserstellung, so stellt die medizinisch-pflegerische Versorgung den Kern der Krankenhausdienstleistung dar. Sie stiftet unmittelbaren Nutzen für externe Kunden (Patienten) und trägt zum strategischen Wettbewerbsvorteil bei. Da eine hierarchische Beziehung zwischen unterstützenden und primären Prozessen besteht, werden die unterstützenden Prozesse von den Kernprozessen dominiert und sind von nur untergeordneter Bedeutung (Rohner 2012). Aufgrund des hochkomplexen und individuellen Charakters sowie des besonderen Stellenwertes medizinischer Leistungserbringung liegt das Potenzial zur klassischen Prozessoptimierung überwiegend in den unterstützenden Prozessen (Zapp und Aleff 2002). Diese müssen dazu 
beitragen, dass die für die Primärleistung notwendigen Ressourcen zum benötigten Zeitpunkt am Einsatzort vorhanden sind und abhängige Teilprozesse reibungslos ablaufen können. Insbesondere Standards und klinische Pfade haben sich als wirksam erwiesen, um Prozesse zu vereinheitlichen sowie zu rationalisieren (Smith und Hillner 2001). Dafür ist es zunächst notwendig, den Weg des Patienten durch das Krankenhaus zu betrachten. Ein Patient durchläuft während des stationären Aufenthaltes mehrere Phasen, die zusammen als sogenannter Patientenpfad bezeichnet werden können. In Anlehnung an den Wertschöpfungsprozess nach Kriegel (2012) lassen sich die in Abbildung 1 dargestellten Phasen des stationären Patientenpfades identifizieren. Farblich hervorgehoben ist dabei der medizinisch-pflegerische Kernprozess, welcher die hauptsächliche Wertschöpfung im Krankenhaus beschreibt.

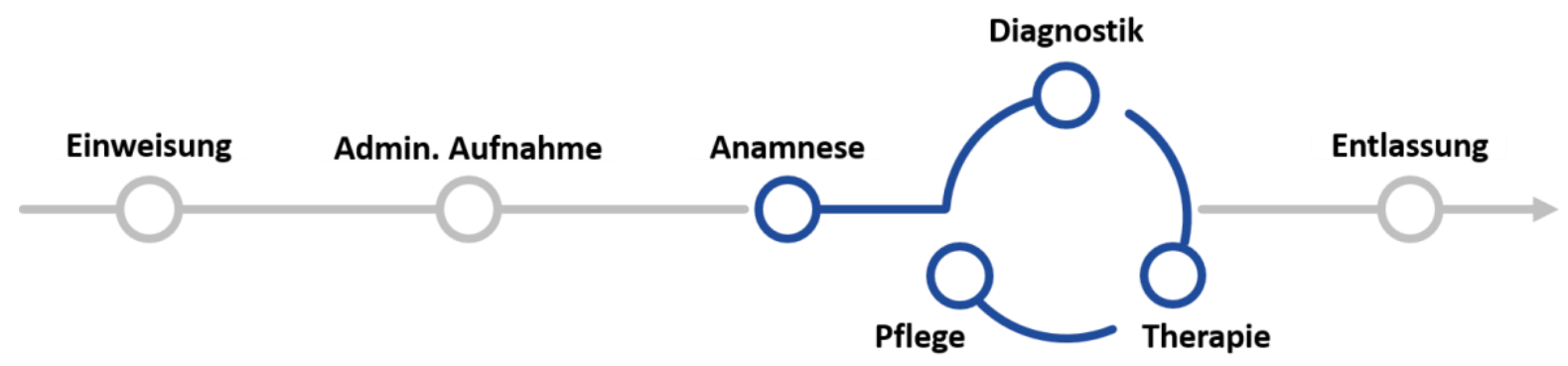

Abbildung 1: Phasen des stationären Wertschöpfungsprozess. Eigene Darstellung in Anlehnung an Kriegel (2012)

Der Patientenpfad umfasst dabei zunächst den Prozessschritt der Einweisung. Diese kann sowohl elektiv, als auch notfallmäßig erfolgen. Die Einweisung ist geprägt von der vorliegenden Indikation zur stationären Behandlung. Der Patient wird bei einer elektiv geplanten Aufnahme mit einem Einweisungsschein vorstellig. Bei einer notfallmäßigen Aufnahme erfolgt der Zugang über die Notaufnahme des Krankenhauses, unter Umständen durch vorangestellten Einsatz des Rettungsdienstes. Nach Urteil des Bundessozialgerichts Kassel (Aktenzeichen B1 KR 26/17 R) ist auch ohne Vorliegen einer Notfallsituation eine eigenständige Einweisung durch den Patienten bei akuter Symptomatik möglich. Als Voraussetzung für die Kostenübernahme gelten dabei jedoch die Zulassung des Krankenhauses sowie die Notwendigkeit und Wirtschaftlichkeit der Behandlung. Im Bereich der Einweisung treten gehäuft Medienbrüche auf, da eine intersektorale Vernetzung noch keine flächendeckende Berücksichtigung findet (Pham et al. 2008). Wie Small et al. (2017) beschreiben, stellt die informationelle Kontinuität der Versorgung einen zentralen Bestandteil effektiven Patienten-Managements dar, dies wird aber oft nicht erreicht, so dass die Patienten die Last der Diskontinuität der Versorgung erleiden oder durch einen Eigenbeitrag kompensieren müssen. Fragmentierung und eine siloartige Kommunikation innerhalb und zwischen den Einrichtungen sind demnach als erhebliche Herausforderungen der Informationskontinuität anzusehen.

Auf die Einweisung folgt die administrative Aufnahme des Patienten. Der Bereich der Patientenaufnahme bildet die zentrale Anlaufstelle für alle Patienten. In diesem Prozessschritt schließen Patient und Krankenhaus einen Behandlungsvertrag. Dazu sind die persönlichen Daten des Patienten, ein Versichertennachweis sowie, falls vorhanden, der Einweisungsschein des vorbehandelnden Arztes erforderlich. Weiterhin bieten sich dem Patienten zu diesem Zeitpunkt Möglichkeiten zum Abschluss von Wahlleistungsverträgen, beispielsweise hinsichtlich der Zimmerbelegung oder dem Wunsch nach Chefarztbehandlung. Ferner werden dem Patienten weitere individuelle Informationsblätter zur Kenntnis und Unterschrift ausgehändigt. Darunter können beispielsweise die Hausordnung, Entgelttarife und Zuzahlungen oder nötige Einwilligungsdokumente zur Datenverarbeitung fallen. Zusätzlich erhält der Patient bei der administrativen Aufnahme seine Patientenidentifikationsmaterialien, häufig 
sind dies Armbänder mit aufgedrucktem Barcode oder appliziertem RFID-Tag. Der Aufnahmeprozess kann nach Rapp (2013) bei mangelhafter Organisation und fehlenden Regeln zu Ineffizienzen und Schnittstellenproblemen führen. Handelt es sich um eine Einweisung im Anschluss an eine Notfallbehandlung, so sind diese administrativen Schritte von nachrangiger Bedeutung, weshalb der Behandlungsvertragsabschluss häufig versäumt wird. Charakteristisch für schlecht organisierte Patientenaufnahmen sind Warteschlangen zu morgendlichen Stoßzeiten, da Elektivpatienten gesammelt einbestellt werden. Abrechnungsrelevante Probleme ergeben sich, insbesondere bei Notfallpatienten, durch fehlende Aufklärung hinsichtlich gewünschter Wahlleistungen. Dies kann dazu führen, dass die erbrachten Leistungen nicht in Rechnung gestellt werden können.

An die administrative Aufnahme schließt sich der medizinisch-pflegerische Kernprozess an. Die dazugehörigen Teilprozesse der Diagnose, Therapie und Pflege bilden einen Kreislauf der ständigen Reflexion und Revision und bedingen sich dadurch gegenseitig (Kriegel 2012).

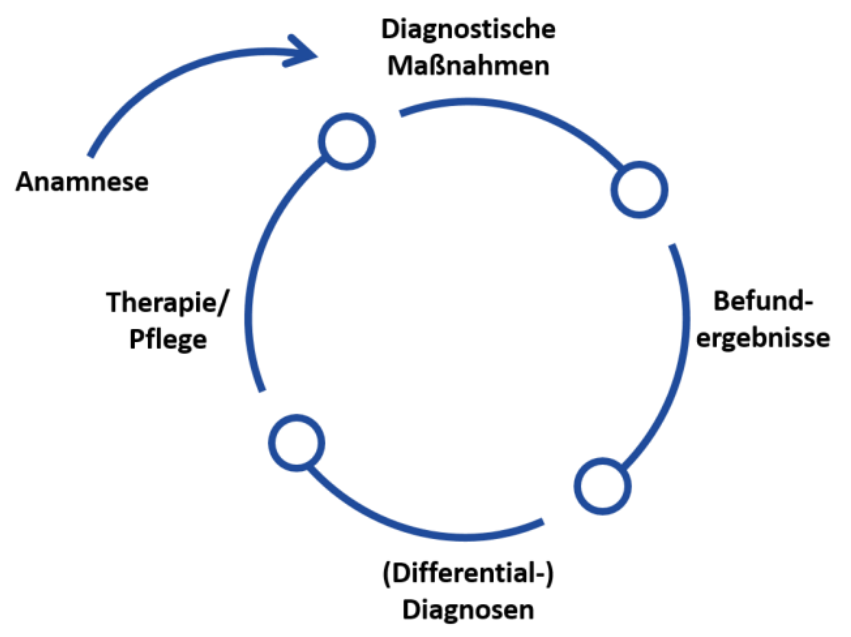

Abbildung 2: Medizinisch-pflegerischer Kernprozess. Eigene Abbildung in Anlehnung an Kriegel (2012)

Der Kreislauf der medizinisch-pflegerischen Leistungserbringung startet mit den erhobenen Daten der Anamnese. Durch gezielte Fragen an den Patienten (Eigenanamnese) oder Dritte (Fremdanamnese) erhalten ärztliches und pflegerisches Personal Auskunft über den gegenwärtigen Gesundheitszustand sowie vergangene Erkrankungen des Patienten. Weiterhin werden bisher erfolgte Behandlungen und bestehende Medikationen sowie die familiäre und soziale Situation abgefragt. Häufig wird diese Abfrage durch standardisierte Anamnesebögen unterstützt. Die Anamnese bildet im Regelfall den aufwändigsten Teil der Erstuntersuchung, welcher zudem noch aufgrund der unterschiedlichen beruflichen Anspruchsgruppen von unausweichlichen Mehrfacherfassungen geprägt ist (Füeß1 und Middeke 2005).

Die Anamnesedaten bilden die Grundlage für die Einleitung diagnostischer Maßnahmen, beispielsweise bildgebende Verfahren oder Laborbestimmungen. Von den dabei objektiv erhobenen Befundergebnissen können in Frage kommende Diagnosen abgeleitet werden. Kommt es bei der Auswertung der Befunde zu mehreren Krankheitsmöglichkeiten, werden so genannte Differentialdiagnosen ermittelt. Unter Differentialdiagnosen versteht man die Berücksichtigung von Erkrankungen mit übereinstimmenden Krankheitsbildern, die ebenfalls als mögliche Ursache für die auftretende Symptomatik bedacht werden müssen. Grundvoraussetzung für das weitere Handeln der Leistungserbringer ist die korrekte Auswahl der Diagnose aus den in Frage kommenden Differentialdiagnosen. Therapietechniken können sowohl konservative (medikamentös/ physikalisch), als auch 
operative Leistungen umfassen und werden durch die medizinischen Berufsgruppen ausgeübt. Es ist unabdingbar, die Arbeitsdiagnose im Verlauf der Therapie fortlaufend zu hinterfragen und Erfolgskontrollen durch weitere diagnostische Maßnahmen vorzunehmen. Sofern im Rahmen dieser Kontrollen kein Therapieerfolg festgestellt werden kann, müssen besagte Differentialdiagnosen hinterfragt und eine dementsprechende Anpassung der eingeleiteten Therapie vorgenommen werden. Im Sinne der Ressourcenschonung sollte bei Anwendung jeglicher Diagnostik ein Augenmerk auf den Mehrwert angeforderter Untersuchungen gelegt werden. Gerade bei einer Vielzahl an Differentialdiagnosen wird Diagnostik häufig unreflektiert ohne zeitliche Ablaufplanung und Sinnhaftigkeit in der Reihenfolge eingesetzt. Insbesondere bei technologischen Innovationen ist diese Vorgehensweise kritisch zu bewerten, da die Anwendung häufig als Zusatz zu bereits erfolgten diagnostischen Maßnahmen zum Zwecke der Befundsicherung erfolgt. So wird beispielsweise erst ein herkömmlicher CT-Scan angefertigt, bevor das qualitativ hochwertigere PET-CT zum Einsatz kommt. Aufgrund anfänglicher Zurückhaltung in der Anwendung neuer Untersuchungs- und Behandlungsmethoden ergibt sich so in der Gesamtsicht ein finanzieller Mehraufwand ohne tatsächlichen diagnostischen Gegenwert. Während des gesamten medizinisch-pflegerischen Kernprozesses begleitet das Pflegepersonal die diagnostischen, therapeutischen, präventiven oder auch rehabilitativen Tätigkeiten des stationären Aufenthaltes. Sie übernehmen die Patientenversorgung in Form medizinischer Betreuung (beispielsweise Messung von Vitalwerten), Assistenz bei ärztlichen Untersuchungen, Verabreichung von Medikamenten, Körperhygiene und Beratung der Patienten. Verbindliche und nachweisbare Dokumentation und Koordination ist dabei ein unerlässlicher Bestandteil der täglichen Aufgaben, um vollständige Informationstransparenz, auch über interne Berufsgruppen und Einzelakteuren hinweg, sicherstellen zu können. Der Fachkräftemangel im Bereich der Pflege verlangt nach Innovationen in ebendiesem Prozessschritt, damit fehlende Personalressourcen durch technologische Unterstützung ausgeglichen werden können. Laut (Schildmann und Voss 2018) betreut eine Fachkraft täglich durchschnittlich 13 Patienten, obgleich der Pflegeschlüssel eine deutlich geringere Anzahl vorgibt, für die Abteilung der Unfallchirurgie beispielsweise zehn Patienten am Tag (Bundesministerium für Gesundheit 2018). Die Bevölkerung scheint entsprechend der Resultate der Umfrage für den Einsatz von digitaler Technik im Kontext der Pflege mehrheitlich offen zu sein. Hauptbestandteil des Diskurses ist, ob der Technikeinsatz in der Pflege, die als menschliche Fürsorgearbeit angesehen ist, den Kern der Leistung negativ verändert und dadurch die Pflegetätigkeit an originärer Bedeutung verliert (Friesacher 2010). In einer von Inverto durchgeführten Studie zur Digitalisierung und technologischen Vernetzung in deutschen Krankenhäusern geht die Mehrheit der Befragten davon aus, dass der Nutzen von Digitalisierungsvorhaben die damit verbundenen Schwierigkeiten bei Weitem übersteigt: 87 Prozent stimmten einer entsprechenden Aussage voll oder überwiegend zu (Kischkewitz 2018). Gegenwärtig ist insbesondere die Digitalisierung der zeitintensiven Dokumentation ein Fokusthema in der Pflege, die patientenbezogene Dokumentation im Krankenhaus hat einen derzeitigen Digitalisierungsgrad von 40 bis 60 Prozent erreicht (Häber et al. 2009). Informationselemente, die als verlässliche Grundlage für alle am Behandlungsprozess Beteiligten dienen, werden häufig noch papierbasiert dokumentiert. Dazu zählen beispielsweise Stammdaten der Patienten, angeforderte und durchgeführte Pflege- und Therapiediagnostik beziehungsweise-Maßnahmen sowie deren Ziele, Vitalparameter und Zustandsbeschreibungen, RisikoAssessments sowie abrechnungsrelevante Leistungsdokumentationen.

Den letzten Schritt des stationären Wertschöpfungsprozesses stellt die Entlassung und Weiterleitung an die Anschlussbehandlung dar. Dafür wird zunächst am Tag der Entlassung eine Abschlussvisite durch das ärztliche und pflegerische Personal durchgeführt. Anschließend händigt der behandelnde Arzt dem Patienten zur 
Weitergabe an den einweisenden Fach- oder Hausarzt in der Regel das papierbasierte Entlassungsschreiben, auch Arztbrief genannt, aus. Dieses Schreiben enthält sämtliche relevanten Informationen über den stationären Aufenthalt, durchgeführte Anamnesen, Diagnostik und Therapien sowie aktuelle Medikation. Die Übermittlung dieser Informationen dient der Sicherstellung des gemeinsamen Wissensstandes aller beteiligten Behandler durch die vollständige Darstellung des Behandlungsverlaufs. Insofern am Entlassungstag noch nicht alle Informationen zur Anfertigung des Arztbriefes vorliegen, wird ein vorläufiger Entlassungsbericht übergeben, welcher die bereits zur Verfügung stehenden Informationen enthält und in Anschluss durch fehlende Befunde und Interpretationen ergänzt wird. Gesetzliche Berücksichtigung findet die Entlassung im Rahmenvertrag Entlass-Management nach $\$ 39$ Abs. 1a S. 9 SGB V, dieser verpflichtet die Einrichtungen zur Organisation für Patienten und Rehabilitanden im Anschluss an ihre stationäre Behandlung. Die Zielsetzung des Rahmenvertrages ist es, die bedarfsgerechte, lückenlose Versorgung der Patienten durch die strukturierte Informationsweitergabe im Anschluss an die Krankenhausbehandlung sicherzustellen. Noch während des stationären Aufenthaltes sind Krankenhäuser in der Verpflichtung, die notwendigen Schritte zur weiterführenden Versorgung einzuleiten, im Bestreben, eine Vernetzung des stationären und ambulanten Versorgungsgeschehens zu ermöglichen. Insbesondere bei komplexen Versorgungssituationen, wie beispielsweise beatmeten Patienten, ist ein erheblicher Koordinationsbedarf unter Einbezug unterschiedlichster Professionen vonnöten. Da das Entlass-Management als Teil der Krankenhausbehandlung angesehen wird, ist für diesen Mehraufwand keine gesonderte Vergütung vorgesehen (Kramer und Schulte-Marin 2017). Dies stellt eine weitere Herausforderung dar, der die Einrichtungen sich trotz ihrer finanziellen Dysbalance stellen müssen.

Bei Betrachtung der einzelnen Schritte des stationären Leistungsprozesses wird die Beteiligung der unterschiedlichen Akteure sowie die bestehende Informationsvielfalt mitsamt ihren Schnittstellenproblemen ersichtlich.

\section{Potenziale durch technologische Innovationen entlang des stationären}

\section{Leistungsprozesses}

Durch gezielten Einsatz digitaler Technologien in jedem Segment des stationären Leistungsprozesses kann es gelingen, die prozessualen Nutzeneinbußen auszugleichen und durch Vernetzung der einzelnen Segmente, in verbesserter Versorgungsqualität, einen Mehrwert für alle Beteiligten zu schaffen.

Betrachtet man insbesondere den Ein- sowie Austritt in beziehungsweise. aus einem Krankenhaus, so wird die Notwendigkeit der intersektoralen Vernetzung offensichtlich. Der stationäre Leistungserbringer ist bei Elektiveinweisungen bezüglich relevanter Befunde in der Regel von denen im Voraus vom einweisenden Arzt erhobenen Daten abhängig. Ebenfalls muss die Nachfolgebehandlung konsistent im Abstimmung mit stationär erhobenen Befunden, erbrachten Leistungen und empfohlenen Therapieansätzen erfolgen. Daher stellt die Integration der Leistungserbringer durch Onlineplattformen eine Möglichkeit dar, medizinische Daten zur Verfügung zu stellen, vorab wie auch im Anschluss an die Krankenhausbehandlung. Zu den Schlüsselmerkmalen dieser sogenannten Einweiserportale gehören die Integration demographischer und klinischer Daten der bestehenden elektronischen Patientenakte, die zentralisierte und fachgebundene Triage von Einweisungen sowie die beidseitig gerichtete Kommunikation zwischen einweisenden Ärzten und stationären Einrichtungen. Technisch betrachtet stellt ein Einweiserportal ein webbasiertes Portal dar, das den Krankenhaussystemen vorgeschaltet ist und den Up- beziehungsweise Download von Daten ermöglicht (Kim et al. 2009). Ein Beispiel für ein derartiges Portal stellt die Telematikplattform der CompuGroup Medical, CGM JESAJANET, dar. Nach Angaben der 
CompuGroup Medical (2019) nutzt jede zweite Arztpraxis in Deutschland bereits heute ein Arztinformationssystem der CGM, weshalb der Aufruf des CGM Einweiserportals ohne erheblichen Aufwand durchgeführt werden kann. Die Kernfunktionen der Anbindungslösung umfassen zum einen die transparente Verfügbarkeit der Daten für den stationären Leistungserbringer vor Stand der Einweisung, damit die Behandlung sorgsam geplant werden kann. Darüber hinaus werden die Daten im Krankenhausinformationssystem kontinuierlich aktualisiert. Auf dieser Grundlage gelingt es, die niedergelassenen Ärzte über Behandlungsverlauf und -Dauer zu informieren und dem Krankenhausaufenthalt nachfolgende Prozesse (Reha, Pflege) zu koordinieren. Auch die Versendung des Arztbriefes an den einweisenden Arzt erfolgt über das Portal. Der aktuell behandelnde Leistungserbringer kann sich so zeitnah vorab und nach Sektorenwechsel des Patienten, von ambulant zu stationär beziehungsweise stationär zu ambulant, über den aktuellen Status bereits durchgeführter Untersuchungen und Behandlungen informieren. Insbesondere Patienten profitieren dabei von der Nutzung des Portals durch ihre behandelnden Ärzte, da folgende Therapien schneller und zielgerichteter eingeleitet werden können. Belastende Doppeluntersuchungen entfallen aufgrund der Transparenz und Verfügbarkeit der Daten, Risiken und potenzielle Wechselwirkungen werden rechtzeitig erkannt und damit die Wahrscheinlichkeit auftretender Fehlbehandlungen vermindert. Zunehmender Beliebtheit erfreuen sich diese Portale insbesondere bei Ärzten, aufgrund der erwarteten Steigerung der Effizienz und Serviceorientierung, da bei Notwendigkeit zur Einweisung Terminreservierungen im Krankenhaus ermöglicht werden. Aber auch die Krankenhausorganisation profitiert von der Nutzung der Portale. Im stationären Behandlungsfall sprechen ambulante Leistungserbringer oftmals subjektive Empfehlungen zur Auswahl des stationären Versorgers an Patienten aus. Dabei bleiben insbesondere Krankenhäuser, die den Vor- und Nachbehandlern verbindliche Informationsströme bieten können, positiv in Erinnerung. Auf diese Weise kann es stationären Leistungserbringern gelingen, die Zuweisertreue zu festigen und dementsprechend auch den kontinuierlichen Patientenstrom zu sichern (Schlegel 2011). Weiterhin bieten sich durch verbindliche und zeitnahe Informationsbereitstellung an die Nachbehandler Potenziale zur Kostenersparnis. Sollten bereits entlassene Patienten aufgrund von Komplikationen einer Wiederaufnahme in das behandelnde Krankenhaus bedürfen, wird diese, sofern sie innerhalb der für die Fallpauschale definierten oberen Grenzverweildauer liegt, als derselbe Fall behandelt. Es erfolgt eine Fallzusammenführung der Aufnahmen. Für Wiederaufnahmen wird, unter der Annahme einer vorab erfolgten „blutigen Entlassung“ (d. h. es wird eine zu frühe Entlassung, vor Beendigung der tatsächlichen stationären Behandlungsbedürftigkeit unterstellt), kein zusätzlicher Erlös für das Krankenhaus generiert. Die zuverlässige Informationsübermittlung an den Fachbeziehungsweise Hausarzt, der die Betreuung im Anschluss an die stationäre Versorgung übernimmt, kann demzufolge zu einer besser abgestimmten ambulanten Nachsorge, der Reduktion von Wiederaufnahmen und damit einhergehend, einer Reduktion der Fallkosten für den stationären Leistungserbringer führen. Dennoch beschreiben Hufnagl et al. (2019), dass zum heutigen Stand Einweiserportale noch keine weitläufige Verbreitung erfahren. Dies ist auf das Fehlen standardisierter Schnittstellen für Autorisierung, Signierung und Verschlüsselung und den damit verbundenen Mehraufwand für die Akteure zurück zu führen (Schlegel 2011).

Auch die administrative Aufnahme bietet durch digitale Transformation Potenziale zur Entlastung des Personals, beispielsweise durch vorgelagerte digitale Aufnahme und Onlineterminvergabe, eine innovative Herangehensweise zur Glättung der aufkommenden Patientenströme. Unter der vorgelagerten digitalen Aufnahme versteht man die Vorab-Berücksichtigung des elektiven Patienten über eine Internetplattform, wie es bereits in diversen Branchen (beispielsweise zur Terminreservierung bei öffentlichen Ämtern) der Regelfall ist. Onlineportale im stationären Gesundheitssetting sollen darüber hinaus für Patienten die Möglichkeit bieten, 
relevante Dokumente bereits vorab hochzuladen und so dem stationären Leistungserbringer zur Verfügung zu stellen. Andererseits sollen es diese Portale aber auch dem Leistungserbringer ermöglichen, Patienten Informationen zur Verfügung zu stellen. Der bestenfalls vollständig automatisierte Versand von Informationsmaterial und Anamnesebögen vor der Einweisung gewährleistet eine bestmögliche Aufnahmevorbereitung. Weiterhin können elektive Behandlungen und nötige Diagnostik bereits in der Terminplanung berücksichtigt, Bettkapazitäten geplant und Wartezeiten vermieden werden. Eine weitere Möglichkeit zur Verkürzung der Wartezeit in der administrativen Aufnahme ergibt sich durch die Bereitstellung von Aufnahme-Terminals, wie sie beim Check-In am Flughafen, beim Erwerb von Fahrttickets im öffentlichen Personenverkehr oder als Geldautomaten zu finden sind. Nach Kucera (2011) betreibt das Klinikum Ingolstadt diese Terminals bereits. Zentrale, im Eingangsbereich der Einrichtung positionierte Self-Serving-Stationen können Teile der Patientenaufnahme in die Verantwortung mündiger und technologieaffiner Patienten, beziehungsweise ihrer Angehörigen, übertragen. Dies spart sowohl patienten-, als auch personalseitig Zeit ein, die Anmeldungsdauer verkürzt sich laut Angaben des Herstellers um bis zu 30 Prozent (Schömann-Finck 2011). Diese Zeitersparnis bietet das Potenzial, wiederum zur Ausführung der patientennahen Prozesse in besserer Versorgungsqualität zur Verfügung zu stehen. Darüber hinaus dient diese aktive Einbindung ebenfalls der Förderung der Autonomie und so der Befähigung und Integration des Patienten in den Versorgungsprozess. Schritte der administrativen Patientenaufnahme, die nicht in den Verantwortungsbereich des Patienten ausgelagert werden können und weiterhin auf Mitarbeiter entfallen, können durch digitale Unterstützung Optimierung erfahren. Eine weitreichende Ressourcenbelastung stellen die bei der administrativen Aufnahme notwendigen Papierdokumente dar. Ziel einer jeden Einrichtung sollte es sein, die Zahl notwendiger Papierdokumente kritisch zu reflektieren und langfristig zu reduzieren. So können kostenintensive Papierarchive abgeschafft und eine durchgängige und nachhaltige Dokumentation ermöglicht werden. Notwendig für die Revisions- und Beweissicherheit digitaler Dokumente ist dabei eine qualifizierte elektronische Signatur gemäß Signaturgesetz und Signaturverordnung, die die handschriftliche Unterschrift nach § 126a BGB Elektronische Form rechtlich ersetzen kann (Roßnagel 2009). Dies kann im Bereich der administrativen Patientenaufnahme erfolgen, indem der Patient die Dokumente auf einem Unterschriften-Tablet digital signiert, welches die biometrische Signatur (beispielsweise Druck und Geschwindigkeit des Schriftbildes) verschlüsselt speichert. Dadurch, dass im Anschluss ein Mitarbeiter mit qualifizierter elektronischer Signatur unterschreibt, wird die Anwesenheit des Patienten bestätigt und das Dokument digital versiegelt.

Der eigentliche medizinisch-pflegerische Kernprozess bietet insbesondere im Bereich der Auftrags- und Befunddokumentation Potenziale zur Digitalisierung. Durch eine elektronische Anamnese können Effizienzreserven gehoben werden, da Anamnesedaten vollumfänglich in höherer Qualität erfasst werden und schnell verfügbar sind (Liu et al. 2012). Diese Erhebung kann zunächst im Rahmen einer Selbstanamnese durch den Patienten erfolgen, bei der Einzelfakten in einer strukturierten Maske, beispielsweise an einem mobilen Endgerät, abgefragt werden. Die individuell abgestimmten Fragebögen und die Eingabesprache können dabei vorab von den Behandlern ausgewählt werden. Diese Art der Anamnese setzt Lese- und Schreibfähigkeit sowie Verständnismöglichkeit voraus. Ergänzt werden muss die Patientenselbstanamnese durch die Anamnese des Arztes, welcher die gegebenen Antworten elektronisch aufarbeitet, eventuell hinterfragt und validiert. Folgende Diagnostik und Therapieeinleitung kann auf Basis verlässlicher und präziser Anamnesedaten zielgerichtet durchgeführt werden, was wiederum zur Schonung von Ressourcen beiträgt. Ergänzt werden diese positiven Aspekte durch die Steigerung der Patientenzufriedenheit und -sicherheit, da die Genauigkeit der Anamnese die 
Reduktion redundanter Diagnostik, welche mitunter für den Patienten belastend sein kann, bedeutet. Nicht nur Doppeluntersuchungen, auch das Vermeiden von Behandlungsfehlern aufgrund fehlerhafter Diagnosestellung stellt derzeit für Patienten eine Schadensquelle dar, die durch eine präzise Anamneseerhebung beseitigt werden kann (Burnett et al. 2011). Die daraus resultierende Zeitersparnis durch den Einsatz elektronischer Anamnese kann in patientenbezogene Tätigkeiten rückgeführt werden und stärkt so wiederum die Arzt-Patienten-Beziehung sowie die Qualität der Versorgung. Eine Weiterentwicklung der elektronischen Anamnese bietet die elektronische Entscheidungsunterstützung in der Diagnostik, welche den Ausschluss möglicher Differentialdiagnosen anhand ihrer Leitsymptome und das Aufstellen von Verdachts- beziehungsweise Arbeitsdiagnosen automatisiert (Hayna und Schmücker 2008). In Diagnostik und Therapie bieten Big Data-Technologien die Möglichkeit, Therapieentscheidungen weitaus fundierter und auf Basis individueller Patientenanforderungen zu treffen. Medizinische Leitlinien und Behandlungsempfehlungen basieren auf klinischen Studien, die ein repräsentatives Patientenklientel, stratifiziert in wenige Hauptgruppen, zur Grundlage haben. Weitaus zukunftsträchtiger und individueller ist die maschinelle Analyse großer patientenbezogener Datenmengen, die vormals noch zu umfassend und heterogen für das menschliche Verständnis waren. Durch algorithmenbasierte Auswertung der Patientendaten können diagnoserelevante Zusammenhänge offengelegt, komplexere Krankheitsprofile erfasst und schließlich präziser und durch die Zielgerichtetheit effizienter diagnostiziert werden (Friele et al. 2018).

Im Bereich konservativer Therapien kann insbesondere die Automatisierung der Arzneimittelgabe dazu beitragen, dass vermeidbare Verschwendung entlang des Versorgungsprozesses eliminiert und so der Beitrag zur Wertschöpfung gesteigert werden kann. Der Prozess der Medikamentenausgabe ist einer der wichtigsten Faktoren für eine sichere Patientenversorgung im Stationsalltag. Häufig belasten patientensicherheitsrelevante Faktoren das Versorgungsumfeld, darunter beispielsweise der hohe Zeitdruck aufgrund der nur begrenzten Personalausstattung, die gleichzeitige Forderung nach rechtzeitiger und umfassender Versorgung einer Vielzahl von Patienten, häufig ohne Verfügbarkeit behandlungsrelevanter Informationen sowie die hohe Anzahl von Medikamenten, die verabreicht und koordiniert werden müssen. Aus diesen Gründen ist die Verbesserung der Systeme und Prozesse, die zur sicheren Abgabe von Medikamenten verwendet werden, von größter Bedeutung. Die Automatisierung der Medikamentenausgabe kann zum einen in den hauseigenen Apotheken erfolgen. Zentralisierte Medikamentenverteilungssysteme umfassen dabei sowohl traditionelle manuelle Dosierungseinheiten als auch stationäre Robotersysteme, die den Prozess der Medikamentenausgabe mit Hilfe der Barcode-Technologie automatisieren. Des Weiteren können im Stationsalltag dezentrale Medikamentenverteilungssysteme, sogenannte Automated Dispensing Cabinets, eingesetzt werden. Zu den Vorteilen einer intelligenten automatisierten Lagerund Ausgabelösung gehört, dass manuelle Arbeitsschritte nicht mehr notwendig sind, das Personal Entlastung erfährt und Patienten die richtige Medikation zuverlässig zur vorgesehenen Zeit erhalten (Fanning et al. 2016). Im Zusammenhang mit operativen Therapiemöglichkeiten stellen insbesondere die Koordination und Dokumentation der Operation unterstützende Prozesse dar, deren Ressourcenaufwand durch digitale Abbildung in erheblichem Maße reduziert werden kann. Checklisten sind in der Luftfahrt ein erprobtes Kontrollinstrument, das zur Erhöhung der Sicherheit beiträgt. Wie im Flugzeugcockpit können auch im Operationssaal Kontrolllisten herangezogen werden (Neumayr et al. 2015). Nach Bauer (2011) sind unerwünschte Vorfälle, wie beispielsweise die Verwechslung von Patienten, Folge multifaktorieller Systemfehler und resultieren nicht alleine aus mangelndem Fachwissen oder dem Fehlverhalten einzelner Akteure. Je mehr Interaktion zwischen den Beteiligten und je mehr anfallende Prozessschritte, desto wahrscheinlicher ist das Auftreten unerwünschter Ereignisse. Im Vordergrund stehen bei der Verwendung von Checklisten der Informationsaustausch aller Beteiligten sowie die Identifizierung 
des Patienten und Transparenz über den beabsichtigten Eingriff. Mit zunehmender Spezialisierung und der damit einhergehenden Arbeitsteilung ist es notwendig, Prozesse in zunehmend interdisziplinärer und interprofes sioneller Weise zu koordinieren, um die Erfüllung spezifizierter Sicherheitsvorgaben zu überwachen sowie die Verteilung von Aufgaben und Verantwortlichkeiten zu regeln. Wie der Hersteller Diagramm Halbach beschreibt, sind digitale OP-Checklisten ebenso effektiv wie die vorherige Papierversion. Der Unterschied besteht jedoch darin, dass die OP-Checklisten während des Schreibens digitalisiert, bei Bedarf automatisch auf Vollständigkeit geprüft, in die elektronische Fallakte eingespeist, mit Zeitstempel versehen und archiviert werden (Diagramm Halbach GmbH 2019). So kann die Effizienz des Prozesses gesteigert und die Informationen nachweislich für alle Beteiligten zur Verfügung gestellt werden.

Mithilfe elektronischer Datenerfassung, beispielsweise durch digitale Visitenwägen oder mobile Eingabegeräte wie Tablet-PCs, sollen diese Elemente digital verfügbar gemacht werden. Informationstransparenz geht mit positiven Erwartungen einher, darunter Zeitersparnis, welche wiederum in patientennahe Zeit investiert werden kann. Weiterhin fallen darunter auch die Vereinfachung und der Transfer bestimmter Aufgaben, Entscheidungsunterstützung, Qualitätssteigerungen bei gleichzeitiger Reduktion der Kosten sowie die Steigerung der Benutzerfreundlichkeit für das Personal (Hielscher et al. 2015; Sowinski et al. 2013). Über die Dokumentation hinaus ergeben sich weitere innovative Ansätze, insbesondere im Bereich der Robotikanwendungen oder durch den Einsatz von Hilfs- und Monitoringsystemen zur Überwachung der Patienten. Hilfsmittel und Monitoringsysteme können bei Pflegetätigkeiten unterstützen, beispielsweise durch den Einsatz von Wearables am Patienten. Diese am Körper zu führenden Geräte bieten das Potenzial, Vitalwerte in Echtzeit zu tracken oder den Aufenthaltsstatus von Patienten transparent zu machen (Delabrida Silva et al. 2017). Auch die sogenannten Unterstützungsprozesse, die in untergeordneter Beziehung zu den medizinisch-pflegerischen Kernleistungen stehen, können durch digitale Technologien effizienter gestaltet werden (Rohner 2012). Beispielhafte Unterstützungsprozesse stellen die Bettenlogistik, Materiallogistik sowie die Speisenversorgung der Patienten dar. All diese Prozesse sind notwendig, um den reibungslosen Ablauf der Kernprozesse zu gewährleisten, welche den direkten Beitrag zur Wertschöpfung (medizinisch-pflegerisches Dienstleistungsgeschehen) liefern. Nach Graf et al. (2013) können die Beteiligten weitere Unterstützung durch Robotik erfahren, so haben sich bereits in vielen deutschen Krankenhäusern Systeme der Service- und Transportrobotik etabliert, die Speisen, patientenindividuell angeforderte Gegenstände (z. B. Zeitschriften), Verbrauchsmaterialien oder Medikamente bereitstellen und die Beseitigung von Wäsche und Abfällen durchführen. Mit Hilfe von drahtlosen Netzwerken, Sensorik und Kameras zur Distanz- und Bewegungserkennung, Scannern sowie Echtzeit-Lokalisierungssystemen (z. B. RFID) wird die autonome Navigation der Roboter sichergestellt. Aufgrund der beträchtlichen Kosten für Anschaffung und Wartung werden Service- und Transportroboter vornehmlich in Einrichtungen mit mehr als 600 Betten eingesetzt. Neben der Unterstützung in Service- und Transport können Robotersysteme auch in der Assistenz des Personals in medizinischen Belangen eingesetzt werden. Sogenannte Assistenzroboter finden in diversen Pilotierungen Einsatz in der Aufklärung und Vorbereitung von Terminen oder im physischen Beistand durch Präsenz nach Operationen, insbesondere bei Demenzerkrankten. Beispielsweise unterstützt der im Rahmen des vom BMBF geförderten Verbundprojektes „SeRoDi - Servicerobotik zur Unterstützung personenbezogener Dienstleistungen“ entwickelte intelligente Pflegewagen das Personal, indem Pflegeutensilien automatisch zur Verfügung gestellt werden. Neben der Bereitstellung der Materialien übernimmt der Roboter, der über mobile Endgeräte an den Einsatzort angefordert werden kann, ebenfalls die Dokumentation des Verbrauchs sowie der damit verbundenen durchgeführten Pflegetätigkeiten (SeRoDi 2019). 


\section{Schlussbetrachtung}

Zur Bewältigung der bestehenden Herausforderungen, denen deutsche Krankenhäuser entgegensehen, bedarf es der Neuausrichtung bestehender Prozessstrukturen. Ziel des Einsatzes von Innovationen ist die Steigerung der Leistungsfähigkeit durch technologische Unterstützung, insbesondere der medizinisch-pflegerischen Tätigkeiten. Der vorliegende Beitrag illustriert, wie eine Innovationsfokussierung entlang der einzelnen Segmente des stationären Leistungsprozesses (Einweisung, Administrative Aufnahme, Anamnese, Diagnostik, Therapie, Pflege, Entlassung) angestrebt werden kann. Das Aufzeigen von Einsatzmöglichkeiten für digitale Lösungen soll den Ablauf bestehender, verschwendungsreicher Arbeitsschritte durch schlanke Technologieinnovationen hinterfragen. Darüber hinaus sollen Gestaltungsanregungen für Prozessstrukturen gegeben werden, die der Steigerung des Wertschöpfungsbeitrages der Einrichtungen und damit der Bewältigung des auftretenden Leistungsvolumens dienen.

Es lässt sich die Schlussfolgerung ziehen, dass die Politik die Verantwortung für die digitale Transformation bisher an die Körperschaften der Selbstverwaltung delegiert hat, was jedoch aufgrund der zum Teil konträren Positionen zur gegenseitigen Blockade und daher Verlangsamung der Entwicklungen führte. Dahingegen existieren auf Mikro- und Mesoebene eine Vielzahl staatlich geförderter, regionaler Pilotprojekte und auch die deutsche Startup Szene beweist, dass innovative Technologien und Anwendungen in der Lage sind, die unterschiedlichen Interessensgruppen des medizinischen Versorgungsprozesses zu unterstützen. Von Seiten der Politik bedarf es der Stärkung der vorgesehenen Führungsrolle und aktiven Gestaltung des digitalen Wandels, unter systematischem Einbezug der Nutzer (Ärzte und Patienten). So kann sich der Branche enormes Entwicklungspotenzial bieten, wobei die Bedeutung der sozialen Interaktion und zwischenmenschlichen Fürsorgearbeit der medizinischen und pflegerischen Berufe Berücksichtigung finden muss.

\section{Kritische Würdigung und Ausblick}

Bisher stellen die Informationssysteme im Krankenhaus größtenteils Insellösungen dar, welche isoliert in ihren einzelnen Anwendungsbereichen genutzt werden. Bildgebende Geräte, Bildarchivierungssysteme oder Kommunikations- und Informationssysteme liegen in der Hand einzelner Fachabteilungen und können nicht zwangsläufig miteinander kommunizieren. Um den reibungslosen Ablauf der beschriebenen Prozessschritte entlang des Patientenpfades gewährleisten zu können, bedarf es der Interoperabilität der Einzelsysteme. Interoperabilität bezeichnet die Fähigkeit von zwei oder mehr Systemen oder Komponenten, Informationen auszutauschen und die Informationen, die ausgetauscht wurden, zu nutzen (Hayes et al. 2000). Diese Fähigkeit muss einerseits technisch vorliegen, damit Daten übertragen werden können. Darüber hinaus muss sie auch prozessual vorliegen, um den Gebrauch der Daten zu gewährleisten. Anbieter von Informationssystemen versuchen, eine modulare Applikationslandschaft vorzuhalten, häufig stellt sogenannte Middleware dabei die Kommunikationslösung dar, Software, die auf einer Übersetzungsebene zwischen Informationssystemen und den darauf aufbauenden Applikationen ausgeführt wird. Ohne die Integration der Insellösungen kann eine Vernetzung der Akteure nicht oder nur unter erheblichem manuellen Aufwand erfolgen, Prozesseffizienzen bleiben unangetastet. Systemverfügbarkeit und Datentransparenz gilt daher als Grundvoraussetzung der getätigten Überlegungen zu Innovationen entlang des stationären Leistungsprozesses in seinen einzelnen Segmenten. Doch nicht nur die technologische Umsetzbarkeit muss gegeben sein, bei der Erbringung von Dienstleistungen im Gesundheitswesen spielt der Faktor Mensch eine tragende Rolle. Insofern die Nutzer der Technologieinnovationen 
deren Verwendungszweck und Sinnhaftigkeit anzweifeln, kann eine Implementierung nicht von Erfolg gezeichnet sein. Sämtliche Innovationen und Veränderungen im Versorgungsalltag müssen daher in enger Abstimmung mit den Beteiligten geplant und schrittweise implementiert werden, damit die Akzeptanz der Technologien sichergestellt werden kann. Die Akzeptanz muss jedoch nicht nur auf Prozessebene geschaffen werden, vielmehr muss den Beteiligten die Furcht genommen werden, in der Zukunft durch Technologien ersetzt zu werden. Ziel der hier beschriebenen Innovationsimpulse ist es diesbezüglich, die Fachkräfte von Tätigkeiten zu entlasten, für die sie eine Überqualifikation besitzen. Eine Technisierung der unterstützenden Prozesse kann so zu einer Steigerung der Möglichkeiten für menschlichen Kontakt führen, zielgerichtet dort, wo Fürsorge und Fachwissen besonders gebraucht wird, der Kernleistung der Versorgung von Patienten. 


\section{Literaturverzeichnis}

Augurzky, Boris; Beivers, Andreas (2014): Krankenhausprivatisierung in Deutschland und ihre Effekte. In: J. Klauber, M. Geraedts, J. Friedrich und J. Wasem (Hg.): Krankenhaus-Report 2014. Schwerpunkt:

Patientensicherheit / mit Online-Zugang zum Internetportal: www.krankenhaus-report-online.de. F.K.:

Schattauer, S. 207-224.

Bauer, Hartwig (2011): Cockpit und OP-Saal: Checklisten verbessern Sicherheit. In: Berlin Medical (01.10), S. 8-12. Online verfügbar unter https://www.dgch.de/fileadmin/media/pdf/dgch/Sicherheitschecklist_Artikel_Bauer.pdf, zuletzt geprüft am 21.05.2019.

Beard, John R.; Bloom, David E. (2015): Towards a comprehensive public health response to population ageing. In: The Lancet 385 (9968), S. 658-661. DOI: 10.1016/S0140-6736(14)61461-6.

Bergmann, Lars; Lacker, Michael (2009): Denken in Wertschöpfung und Verschwendung. In: Uwe Dombrowski, Christoph Herrmann, Thomas Lacker und Sabine Sonnentag (Hg.): Modernisierung kleiner und mittlerer Unternehmen. Ein ganzheitliches Konzept. Berlin, Heidelberg: Springer (VDI-Buch), S. 161-168. Online verfügbar unter https://doi.org/10.1007/978-3-540-92927-7_9.

Bundesministerium für Gesundheit (2018): Pflegepersonaluntergrenzen. Online verfügbar unter https://www.bundesgesundheitsministerium.de/personaluntergrenzen.html.

Bundesministerium für Wirtschaft und Energie (2018): Monitoring-Report Wirtschaft DIGITAL 2018 Wirtschaftsindex DIGITAL. Hg. v. Bundesministerium für Wirtschaft und Energie. Online verfügbar unter https://www.bmwi.de/Redaktion/DE/Publikationen/Digitale-Welt/monitoring-report-wirtschaft-digital-2018kurzfassung.pdf?_blob=publicationFile \&v=22.

Burnett, Susan J.; Deelchand, Vashist; Franklin, Bryony Dean; Moorthy, Krishna; Vincent, Charles (2011): Missing clinical information in NHS hospital outpatient clinics: prevalence, causes and effects on patient care. In: BMC Health Services Research 11, S. 114. DOI: 10.1186/1472-6963-11-114.

CompuGroup Medical (2019): CGM JESAJANET auf einen Blick. Online verfügbar unter https://www.cgm.com/de/kis/zuweiserportal/cgm_jesajanet/cgm_jesajanet_das_produkt/das_produkt.de.jsp.

Deiters, W.; Burmann, A.; Meister, S. (2018): Digitalisierungsstrategien für das Krankenhaus der Zukunft. In: Der Urologe. Ausg. A 57 (9), S. 1031-1039. DOI: 10.1007/s00120-018-0731-2.

Delabrida Silva, S. E.; Rabelo Oliveira, R. A.; Loureiro, A.A.F. (2017): Examining Developments and Applications of Wearable Devices in Modern Society: IGI Global. Online verfügbar unter https://books.google.de/books?id=gBgxDwAAQBAJ.

Deloitte (2018): Krankenhaus Rating Report 2018: Fachkräftemangel als größte Herausforderung für die Gesundheitswirtschaft. Online verfügbar unter https://www2.deloitte.com/content/dam/Deloitte/de/Documents/life-sciences-health-care/Deloitte-LSHCInfografik-Krankenhau-Report-2018.pdf, zuletzt geprüft am 02.02.2018.

Denner, Marie-Sophie; Püschel, Louis Christian; Röglinger, Maximilian (2018): How to Exploit the Digitalization Potential of Business Processes. In: 1867-0202.

Di Vincenzo, Fausto (2018): Exploring the networking behaviors of hospital organizations. In: BMC Health Services Research 18 (1), S. 334. DOI: 10.1186/s12913-018-3144-4.

Diagramm Halbach GmbH (2019): OP-Protokolle / WHO-Checklisten. Online verfügbar unter https://www.propatientensicherheit.de/produkte/mobile-dokumentation-mit-digitalem-papier/op-protokolle-who-checklisten/.

Fanning, Laura; Jones, Nick; Manias, Elizabeth (2016): Impact of automated dispensing cabinets on medication selection and preparation error rates in an emergency department: a prospective and direct observational beforeand-after study. In: Journal of evaluation in clinical practice 22 (2), S. 156-163. DOI: 10.1111/jep.12445.

Friele, Minou; Jannes, Christiane; Jannes, Marc; Woopen, Christian (2018): Algorithmen in der digitalen Gesundheitsversorgung. Eine interdisziplinäre Analyse. Bertelsmann Stiftung. Gütersloh. Online verfügbar unter 
https://www.bertelsmann-

stiftung.de/fileadmin/files/BSt/Publikationen/GrauePublikationen/VV_Studie_Algorithmen.pdf.

Friesacher, Heiner (2010): Pflege und Technik - eine kritische Analyse. In: Pflege\& Gesellschaft 15 (4), S. 293313.

Füeßl, Hermann; Middeke, Martin (2005): Anamnese und klinische Untersuchung. Mit CD-ROM Herz- und Lungenauskultation. 3., überarb. Aufl. Stuttgart: Thieme (Duale Reihe).

Garcia, Andrea; Revere, Lee; Sharath, Sherene; Kougias, Panos (2017): Implications of Clinical Documentation (In)Accuracy: A Pilot Study Among General Surgery Residents. In: Hospital topics 95 (2), S. 27-31. DOI: 10.1080/00185868.2017.1300471.

Georgantzas, Nicholas C.; Katsamakas, Evangelos G. (2008): Information systems research with system dynamics. In: System Dynamics Review 24 (3), S. 247-264. DOI: 10.1002/sdr.420.

Gerlinger, Thomas (2013): Integrationsbedarf und Integrationsbemühungen. Bundeszentrale für politische Bildung. Online verfügbar unter http://www.bpb.de/politik/innenpolitik/gesundheitspolitik/156022/integrationsbedarf-undintegrationsbemuehungen.

Goedereis, Klaus (2009): Einfluss auf die Versorgungslandschaft. In: Ferdinand Rau, Norbert Roeder und Peter Hensen (Hg.): Auswirkungen der DRG-Einführung in Deutschland. Standortbestimmung und Perspektiven. 1. Aufl. s.l.: Kohlhammer Verlag, S. 239-253.

Graf, B.; Heyer, T.; Klein, B.; Wallhoff, F. (2013): Servicerobotik für den demografischen Wandel. Mögliche Einsatzfelder und aktueller Entwicklungsstand. In: Bundesgesundheitsblatt - Gesundheitsforschung Gesundheitsschutz 56 (8), S. 1145-1152. DOI: 10.1007/s00103-013-1755-9.

Häber, Anke; Dujat, Carl; Schmücker, Paul (2009): Elektronische Dokumenten- und Archivmanagement der Gegenwart und Zukunft? In: mdi - Forum der Medizin_Dokumentation und Medizin_Informatik, S. 35.

Hayes, J. G.; Peyrovian, E.; Sarin, S.; Schmidt, M.-T.; Swenson, K. D.; Weber, R. (2000): Workflow interoperability standards for the Internet. In: IEEE Internet Comput. 4 (3), S. 37-45. DOI: $10.1109 / 4236.845389$.

Hayna, Steffen; Schmücker, Paul (2008): eAnamnese, ein einrichtungs-, sektoren- und berufsgruppenübergreifender Lösungsansatz für die Elektronische Anamnese. In: Achim Jäckel (Hg.): Telemedizinführer 2009. 10, veränd. Aufl. Darmstadt: Minerva, S. 183.

Hielscher, Volker; Nock, Lukas; Kirchen-Peters, Sabine (2015): Technikeinsatz in der Altenpflege. Potenziale und Probleme in empirischer Perspektive. Baden-Baden: Nomos (Forschung aus der Hans-Böckler-Stiftung).

Hufnagl, Christine; Doctor, Eileen; Behrens, Laura; Buck, Christoph; Eymann, Torsten (2019): Digitisation along the Patient Pathway in Hospitals. In: Research Papers. Online verfügbar unter https://aisel.aisnet.org/ecis2019_rp/193.

Kim, Yeuen; Chen, Alice Hm; Keith, Ellen; Yee, Hal F.; Kushel, Margot B. (2009): Not perfect, but better: primary care providers' experiences with electronic referrals in a safety net health system. In: Journal of general internal medicine 24 (5), S. 614-619. DOI: 10.1007/s11606-009-0955-3.

Kischkewitz, Jan-Christoph (2018): Heime und Spitäler: Der weite Weg zum Krankenhaus 4.0. Hg. v. Inverto $\mathrm{GmbH}$. Online verfügbar unter https://www.inverto.com/publikationen/23-03-18-heime-und-spitaeler-der-weiteweg-zum-krankenhaus-4-0/.

Kramer, Ulrike; Schulte-Marin, Veronika (2017): Allen Anforderungen gewachsen. In: f\&w führen und wirtschaften im Krankenhaus (8), S. 730.

Kriegel, Johannes (2012): Krankenhauslogistik. Innovative Strategien für die Ressourcenbereitstellung und Prozessoptimierung im Krankenhauswesen. Wiesbaden: Gabler Verlag. Online verfügbar unter http://dx.doi.org/10.1007/978-3-8349-3648-6. 
Kucera, Martin (2011): Maschine begrüßt Patienten. kma Online. Online verfügbar unter https://www.kmaonline.de/aktuelles/it-technik/detail/maschine-begruesst-patienten-a-21933.

Liu, Chia-Hui; Chung, Yu-Fang; Chen, Tzer-Shyong; Wang, Sheng-De (2012): Mobile agent application and integration in electronic anamnesis system. In: Journal of medical systems 36 (3), S. 1009-1020. DOI: 10.1007/s10916-010-9563-3.

Lohmann, Heinz (2009): Erfolgsfaktor Medizin: Anforderungen an ein modernes Krankenhausmanagement. In: Ingo Behrendt, Hans-Joachim König und Ulrich Krystek (Hg.): Zukunftsorientierter Wandel im Krankenhausmanagement. Outsourcing, IT-Nutzenpotenziale, Kooperationsformen, Changemanagement. Berlin, Heidelberg: Springer-Verlag Berlin Heidelberg, S. 3-13.

Neumayr, A.; Baubin, M.; Schinnerl, A. (2015): Risikomanagement in der prähospitalen Notfallmedizin: Werkzeuge, Maßnahmen, Methoden: Springer Berlin Heidelberg. Online verfügbar unter https://books.google.de/books?id=raEvCwAAQBAJ.

Pham, Hoangmai H.; Grossman, Joy M.; Cohen, Genna; Bodenheimer, Thomas (2008): Hospitalists and care transitions: the divorce of inpatient and outpatient care. In: Health affairs (Project Hope) 27 (5), S. 1315-1327. DOI: 10.1377/hlthaff.27.5.1315.

Rapp, Boris (2013): Fallmanagement im Krankenhaus. Grundlagen und Praxistipps für erfolgreiche Klinikprozesse. 1. Aufl. s.l.: Kohlhammer Verlag. Online verfügbar unter http://gbv.eblib.com/patron/FullRecord.aspx?p=1714515.

Rau, Ferdinand; Roeder, Norbert; Hensen, Peter (2009): Zum Stand der deutschen DRG-Einführung: Erkenntnisse, Erfahrungen und Meinungen. In: Ferdinand Rau, Norbert Roeder und Peter Hensen (Hg.): Auswirkungen der DRG-Einführung in Deutschland. Standortbestimmung und Perspektiven. 1. Aufl. s.1.: Kohlhammer Verlag, S. 9-22.

Riepe, Claudia; Schwanenflügel, Matthias von (2013): Ethische Herausforderungen und Chancen von Telematik und Telemedizin. In: GuS 67 (4), S. 52-54. DOI: 10.5771/1611-5821-2013-4-52.

Rohner, Peter (2012): Achieving impact with clinical process management in hospitals: an inspiring case. In: Business Process Mgmt Journal 18 (4), S. 600-624. DOI: 10.1108/14637151211253756.

Roßnagel, H. (2009): Mobile qualifizierte elektronische Signaturen: Analyse der Hemmnisfaktoren und Gestaltungsvorschläge zur Einführung: Gabler Verlag. Online verfügbar unter https://books.google.de/books?id=80u5uRf8kPQC.

Rynning, Elisabeth (2008): The Ageing Populations of Europe - Implications for Health Systems and Patients' Rights. In: European Journal of Health Law 15 (3), S. 297-306. DOI: 10.1163/157180908X338241.

Schildmann, Christina; Voss, Dorothea (2018): Aufwertung von sozialen Dienstleistungen. Warum sie notwendig ist und welche Stolpersteine noch auf dem Weg liegen. Hg. v. Hans-Böckler-Stiftung. Düsseldorf (Forschungsförderung Report Nr. 4). Online verfügbar unter https://www.boeckler.de/pdf/p_fofoe_report_004_2018.pdf, zuletzt geprüft am 21.05.2019.

Schlegel, Helmut (2011): Das Einweiserportal - ein ausgemachtens Spannungsfeld. Erwartungshaltungen zwischen Kosten und Qualität. In: Krankenhaus-IT Journal (6), S. 14.

Schömann-Finck, Stefanie (2011): Self-Check-in im Krankenhaus. Invidis. Online verfügbar unter https://invidis.de/2011/02/self-check-in-im-krankenhaus/.

SeRoDi (2019): Projektschwerpunkte. Online verfügbar unter https://www.serodi.de/?page_id=37.

Simon, Michael (2014): Ökonomisierung und soziale Ungleichheit in Organisationen des Gesundheitswesens. In: Alexandra Manzei und Rudi Schmiede (Hg.): 20 Jahre Wettbewerb im Gesundheitswesen. Wiesbaden: Springer Fachmedien Wiesbaden, S. 157-177.

Small, Serena; Peddie, David; Ackerley, Christine; Hohl, Corinne; Balka, Ellen (2017): Patient experiences with informational discontinuity of care: Bridging the gaps. In: Procedia Computer Science 113, S. 295-302. DOI: 10.1016/j.procs.2017.08.327. 
Smith, T. J.; Hillner, B. E. (2001): Ensuring quality cancer care by the use of clinical practice guidelines and critical pathways. In: Journal of clinical oncology : official journal of the American Society of Clinical Oncology 19 (11), S. 2886-2897. DOI: 10.1200/JCO.2001.19.11.2886.

Sowinski, Christine; Kirchen-Peters, Sabine; Hielscher, Volker (2013): Praxiserfahrungen zum Technikeinsatz in der Altenpflege. Hg. v. Kuratorium Deutsche Altershilfe. Köln. Online verfügbar unter https://www.boeckler.de/pdf_fof/91394.pdf.

Springer Medizin Pflegezeitschrift (2018): Krankenhaus Rating Report 2018. Fachzeitschrift für stationäre und ambulante Pflege. In: Pflegezeitschrift 71 (8), S. 7. DOI: 10.1007/s41906-018-0613-7.

Tebroke, Ev (2017): Telematik-Infrastruktur. Die Basis für den Rollout steht. Online verfügbar unter https://www.pharmazeutische-zeitung.de/ausgabe-232017/die-basis-fuer-den-rollout-steht/.

Tetzlaff, Juliane; Muschik, Denise; Epping, Jelena; Eberhard, Sveja; Geyer, Siegfried (2017): Expansion or compression of multimorbidity? 10-year development of life years spent in multimorbidity based on health insurance claims data of Lower Saxony, Germany. In: International journal of public health 62 (6), S. 679-686. DOI: $10.1007 / \mathrm{s} 00038-017-0962-9$.

Traeger, D. H. (2013): Grundgedanken der Lean Production: Vieweg+Teubner Verlag. Online verfügbar unter https://books.google.de/books?id=IajvBgAAQBAJ.

van Baal, Pieter; Morton, Alec; Meltzer, David; Brouwer, Werner (2018): Future unrelated medical costs need to be considered in cost effectiveness analysis. In: The European Journal of Health Economics. DOI:

10.1007/s10198-018-0976-0.

Weiß, R. (2014): Das Aufgabenspektrum der Pflege: Eine kritische Studie aus der pflegewissenschaftlichen und betriebswirtschaftlichen Sichtweise: Diplomica Verlag. Online verfügbar unter

https://books.google.de/books?id=IRmwBQAAQBAJ.

Weissman, Joel S.; Rothschild, Jeffrey M.; Bendavid, Eran; Sprivulis, Peter; Cook, E. Francis; Evans, R. Scott et al. (2007): Hospital workload and adverse events. In: Medical care 45 (5), S. 448-455. DOI: 10.1097/01.mlr.0000257231.86368.09.

Zapp, Winfried; Aleff, Gregor (Hg.) (2002): Prozessgestaltung im Krankenhaus. Heidelberg: Economica-Verlag (Wirtschaft in der Praxis). 